\title{
Formación crítica en medicina
}

\author{
Eduardo García-Soblechero, Rocío C. Martín-García de Arboleya
}

Habiendo leído con interés el artículo de MirónCanelo et al, publicado recientemente en Educación Médica [1], parece conveniente realizar algunas consideraciones. Aunque muchos de sus resultados pueden reflejar la realidad existente en las aulas y hospitales donde se imparte la docencia en medicina, algunas de las conclusiones planteadas debemos tomarlas con cautela.

Debido al diseño del estudio, de carácter transversal a través de una encuesta, algunas afirmaciones podrían explicarse por un sesgo en la selección de los encuestados [2]. Según se contempla en la sección de 'sujetos y métodos', la encuesta se realiza un día de clase al azar dentro del último mes lectivo. Debemos advertir que la elección de 'un día al azar' no garantiza la 'aleatoriedad' de nuestra muestra y, por tanto, su representatividad puede quedar afectada. El 70\% de los alumnos (225 alumnos) matriculados completa la encuesta, pero nunca sabremos lo que hubiera ocurrido con el otro 30\% (96 alumnos) si también la hubiera realizado. Sólo con técnicas específicas de muestreo aleatorio podríamos haber obtenido una muestra representativa de la población diana (estudiantes de sexto curso de medicina).

Fijándonos en la variable objetivo 'fuente de conocimiento utilizado como apoyo en su formación de pregrado', no sorprende el alto índice de utilización de los 'apuntes de clase', ya que la encuesta se realiza precisamente entre los alumnos que asisten a clase. Este es el problema de utilizar una muestra no aleatorizada: si un alto porcentaje de alumnos que no cumplimentaron la encuesta por no asistir a clase utilizara otra fuente de conocimiento, los resultados habrían podido ser bastante diferentes. Esta situación parecería incluso lógica, ya que podría ser que los alumnos que no acuden a clase por utilizar otras fuentes de conocimiento tampoco se hallaran en clase el día de la realización de la encuesta.

Por último, analizando los resultados de otra de las variables de estudio objetivo, la 'opinión sobre si el pregrado está condicionado por el examen MIR', debemos tener en cuenta la posible existencia de un sesgo histórico o de memoria que podría modificar la relación encontrada [2,3]. Desde que la Unión Eu- ropea exigió en el año 1995 al menos dos años de formación posgraduada a los licenciados de medicina para trabajar en la sanidad pública, el sistema MIR se ha erigido en España como el único camino desde ese año para poder realizar dicha formación posgrado en nuestro país. Este hecho podría haber influido en que la opinión de que la formación pregrado estaba muy condicionada por el examen MIR hubiera calado más profundo en una población, la de los estudiantes de 1999, que tenía aún reciente el recuerdo de la resolución europea que modificaba el sistema anterior y adjudicaba un papel principal al sistema MIR. Sin embargo, es posible que entre los años 2008-2010 se hubiera amortiguado ese efecto de la importancia del sistema MIR como innovador y modificador de la formación de pregrado. Por tanto, es difícil discernir cuánta culpa tiene este posible efecto sobre los resultados reflejados en esta variable del estudio.

Como expresa el artículo de Mirón-Canelo et al [1], tal vez la utilización de revistas actualizadas en el conocimiento médico sea escasa entre nuestros estudiantes de medicina. Creemos que se precisa una formación previa (idealmente en los primeros años de pregrado) en búsqueda bibliográfica y lectura crítica de artículos sanitarios de calidad, algo que parece obligado con el emerger actual de la medicina basada en la evidencia.

El sistema de formación de pregrado en medicina necesita evolucionar acorde con la sociedad actual y, con ello, su método de evaluación. Tal vez se precise una reforma del examen MIR. Muy probablemente haya que virar hacia un método formativo que prepare para un tipo de asistencia según un método biopsicosocial, fundiendo la capacidad técnica y la humana en el arte de atender al paciente.

\footnotetext{
Bibliografía

1. Mirón-Canelo JA, Iglesias-De Sena H, Alonso-Sardón M. Valoración de los estudiantes sobre su formación en la Facultad de Medicina. Educ Med 2011; 14: 221-8.

2. Argimón JM, Jiménez J. Métodos de investigación clínica y epidemiológica. Barcelona: Elsevier; 2009.

3. Hernández B, Velasco-Mondragón HE. Encuestas transversales. Salud Publica Mex 2000; 42: 447-55.
}

UGC Pediatría (E. García-Soblechero); UGC Cirugía General y del Aparato Digestivo (R.C. Martín-García de Arboleya); Hospital Juan Ramón Jiménez. Huelva, España..

Correspondencia:

Dr. Eduardo García Soblechero. UGC Pediatría. Hospital Juan Ramón Jiménez. Ronda Norte, s/n. E-21005 Huelva.

E-mail:

edusoblechero@hotmail.com

(c) 2012 Educación Médica 